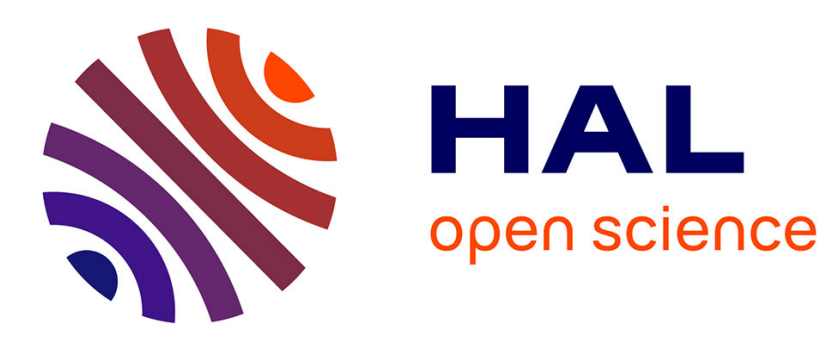

\title{
AColDPS : Robust and Unsupervised Automatic Color Document Processing System
}

Louisa Kessi, Frank Le Bourgeois, Christophe Garcia, Jean Duong

\section{To cite this version:}

Louisa Kessi, Frank Le Bourgeois, Christophe Garcia, Jean Duong. AColDPS: Robust and Unsupervised Automatic Color Document Processing System . VISAPP 2015, 10th International Joint Conference on Computer Vision, Imaging and Computer Graphics Theory and Applications, Mar 2015, Berlin, Germany. 10.5220/0005315801740185 . hal-01272989

\section{HAL Id: hal-01272989 \\ https://hal.science/hal-01272989}

Submitted on 16 Feb 2016

HAL is a multi-disciplinary open access archive for the deposit and dissemination of scientific research documents, whether they are published or not. The documents may come from teaching and research institutions in France or abroad, or from public or private research centers.
L'archive ouverte pluridisciplinaire HAL, est destinée au dépôt et à la diffusion de documents scientifiques de niveau recherche, publiés ou non, émanant des établissements d'enseignement et de recherche français ou étrangers, des laboratoires publics ou privés. 


\section{AColDPS :Robust and Unsupervised Automatic Color Document Processing System}

CONFERENCE PAPER - MARCH 2015

CITATION

1

4 AUTHORS, INCLUDING:

Kessi Louisa

Institut National des Sciences Appliquées d.

3 PUBLICATIONS 1 CITATION

SEE PROFILE

\section{Christophe Garcia}

Institut National des Sciences Appliquées d...

143 PUBLICATIONS $\quad 2,024$ CITATIONS

SEE PROFILE
READS

98

\section{Frank Lebourgeois}

Institut National des Sciences Appliquées d...

79 PUBLICATIONS 455 CITATIONS

SEE PROFILE 


\title{
ACOLDPS: Robust and Unsupervised Automatic Color Document Processing System
}

\author{
Louisa Kessi ${ }^{1,2}$, Frank Lebourgeois ${ }^{1,2}$ Christophe Garcia ${ }^{1,2}$ and Jean Duong ${ }^{1.2}$ \\ ${ }^{1}$ Université de Lyon, CNRS \\ ${ }^{2}$ INSA-Lyon, LIRIS, UMR5205,F-69621, France \\ \{louisa.kessi,franck.lebourgeois,christophe.garcia, jean.duong\}@ liris.cnrs.fr
}

Keywords: Document image analysis, color processing, business document, mathematical morphology, color morphology.

Abstract: This paper presents the first fully automatic color analysis system suited for business documents. Our pixelbased approach uses mainly color morphology and does not require any training, manual assistance, prior knowledge or model. We developed a robust color segmentation system adapted for invoices and forms with significant color complexity and dithered background. The system achieves several operations to segment automatically color images, separate text from noise and graphics and provides color information about text color. The contribution of our work is Tree-fold. Firstly, it is the usage of color morphology to simultaneously segment both text and inverted text. Our system processes inverted and non-inverted text automatically using conditional color dilation and erosion, even in cases where there are overlaps between the two. Secondly, it is the extraction of geodesic measures using morphological convolution in order to separate text, noise and graphical elements. Thirdly, we develop a method to disconnect characters touching or overlapping graphical elements. Our system can separate characters that touch straight lines, split overlapped characters with different colors and separate characters from graphics if they have different colors. A color analysis stage automatically calculates the number of character colors. The proposed system is generic enough to process a wide range of images of digitized business documents from different origins. It outperforms the classical approach that uses binarization of greyscale images.

\section{INTRODUCTION}

Color document processing is an active research area with significant applications. In recent years, there has been an increasing need for systems which are able to convert pre-printed color documents into digital format automatically. Most of the time, the color image is converted into a greyscale image. However, the performance decreases when the segmentation fails. Nowadays, companies have to deal with huge volumes of administrative color documents such as invoices, forms, and letters and so on. Indeed, some companies can have to cope with dithering documents, complex color background and linear color variations, which amounts to not knowing if text is darker or lighter compared to the background, highlighting regions, corrective red overload on black text and not uniform color text/graphics overlapping. Indeed, some dithered documents may not lead to a correct automatic analysis. Smoothing most often permits to reduce dithering significantly but can also seriously damage the text. Therefore, the color information is significant. Then, a color-based segmentation could improve the process.
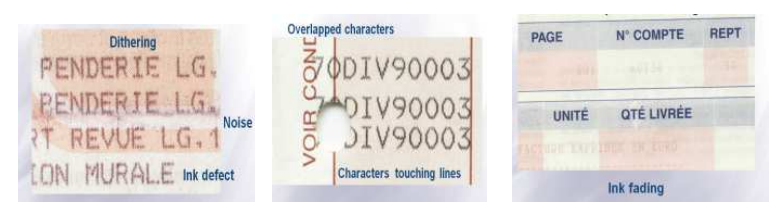

Figure 1: Issues some examples of several difficulties presented in color business document.

\subsection{Related Work}

As far as the authors know, there are only few works about the use of the color for document analysis. The only referenced work for color documents come 
from (Bottou, 2001) for the $\mathrm{DjVu}$ compression. In the work of (Karatzas et al., 2007), (Y. Peng et al., 2007) and (Jung et al., 2004) localization of colored text is described. OCR of colored characters is presented in (Badekas et al., 2006). Most of the research on color documents considers the classification as an essential pre-processing step to any analysis. This facilitates the extraction of the connected components by a simple growing region. The work focuses mostly on-pixel classification approaches to reduce the number of colors found. The pixel classification consists of assigning each pixel of the image to the color class layer according to its colorimetric appearance. In this context, (Ouji et al., 2011) introduce a new pseudo-saturation measure to separate color layers and monochrome layer. The author segments text colors and background by selecting maxima in the hue histogram. However, this global analysis of the image cannot make the difference between text colors and background color. Moreover it works only for cleaned images. This method is not operating for business document images. (Ait Younes et al., 2005) describe the color thanks to fuzzy sets in order to classify images based on dominant colors. However, they dealing with a fixed number of colors which is not optimized for documents containing just a few colors.(Carel et al., 2013) propose a hierarchical clustering based approach to extract dominant color masks of administrative documents. Moreover, this approach was evaluated on a relatively small base. Therefore, they need to provide more extensive quantitative analysis of the process both in terms of its effectiveness and computational requirements.

On the other hand, this method requires user interaction for setting threshold parameters order to decide what a dominant color is or not. This approach works only for a specific category of images and is very sensitive for noises. This paper addresses the most challenging problem of color morphology. We use this theory to develop the first robust unsupervised Automatic Color Document Processing System pixel-based approach.

\subsection{Motivation}

In this paper, we present an automatic system which segments color characters from business documents and especially from forms and invoices. Our objective is achieved if all color characters are correctly segmented from the background, using an automatic procedure without any information provided by the user. Contrary to previous works, we develop the first full data-driven pixel-based approach which doesn't need any priori information such as the number of text colors or any training or manual assistance. The paper is organized as follows. Section 2 presents the color morphology fundamentals and in Section 3 there is a detailed description of our approach. Results are discussed in Section 4. In Section 5 conclusions are drawn.

\section{COLOR MATHEMATICAL MORPHOLOGY}

The theory of Mathematical Morphology (Serra, 1982) consists of quantitatively describing the geometric structures present in the image, and offers a wide range of tools (Soille, 2004). Motivated by the recent researches on extension of morphological operations to color images(Chanussot et al. 1998), we have decided to employ color mathematical morphology tools to achieve color document images segmentation in a flexible and fast manner. Our system uses the morphological convolution (1) and the lexicographic order to encode color vectors into a scalar.The morphological convolution transforms a binary image $I$ into an image $I_{G}$ by taking the largest or smallest value of $I+V$ on the domain of definition $D v$ of a neighborhood $V$.

$\mathrm{I}_{\mathrm{G}}(\mathrm{x})=\operatorname{Min}$ or $\operatorname{Max}\{\mathrm{I}(\mathrm{x}+\mathrm{k})+\mathrm{V}(\mathrm{k})\} \forall \mathrm{k} \in \mathrm{D}_{\mathrm{V}} \forall \mathrm{x} \in \mathrm{X}$ (1)

By using the interleaved bits, color morphology in 3 dimensional spaces can be substituted efficiently by a scalar classical morphology (Chanussot et al.1998) popularized by (Aptoula et al., 2009) show that the scalar morphology by using the encoding scheme illustrated in Figure 2 avoids pseudo colors apparition. We reduce the dissymmetry between color components by rotating the sequence of RGB.

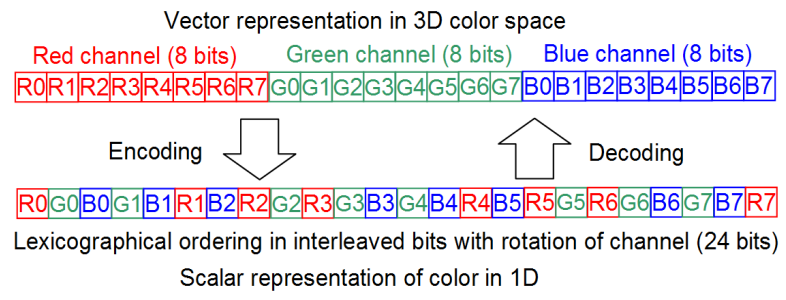

Figure 2: Color coding in scalar by using interleaved bit and rotation of the sequence of RGB.

\section{OUR PROPOSITION}

Figure 3 illustrates the main steps of the proposed system. Each step will be detailed in the next sections following the organisation of the paper. 


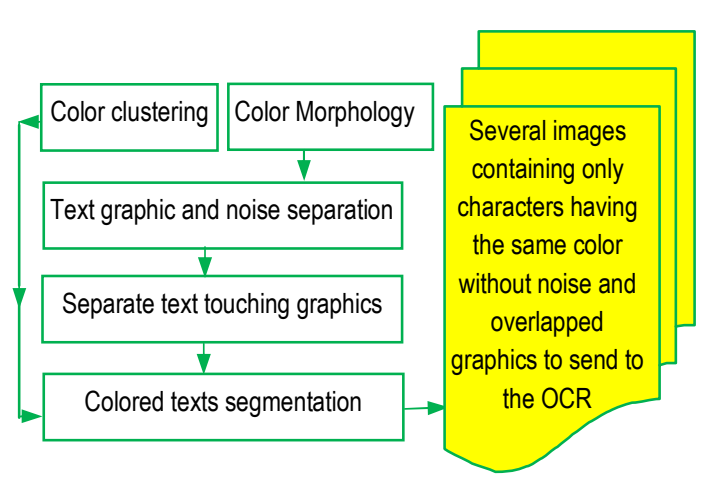

Figure 3: illustrates the overall scheme of AColDPS.

\subsection{Color Clustering}

The spatial MeanShift originally introduced by (Comaniciu et al., 2002) demonstrates that a densitybased clustering is efficient for color clustering and reduces the complexity of the original MeanShift algorithm by introducing the spatial coordinates into the color vector. Applied to color documents, the spatial Mean Shift requires a complex fusion step to restore the continuity of the characters. The Fast Integral MeanShift proposed by (Lebourgeois et al., 2013), reduces the complexity of the original MeanShift from $\mathrm{O}\left(\mathrm{N}^{2}\right)$ to $\mathrm{O}(\mathrm{N})$. The global MeanShift can be used now on large datasets and especially on color images of documents in high resolution. AColDPS uses the Fast Integral MeanShift to reduce the number of colors of an image of 2492 x 3558 in 300 dpi to a very reduced number of colors in less than $3 \mathrm{sec}$. This clustering is robust to large change of the colors of the characters.

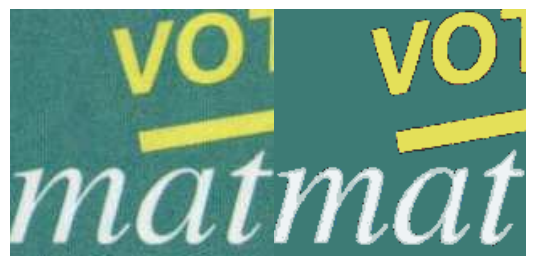

a) Color transition detected by the Mean Shift

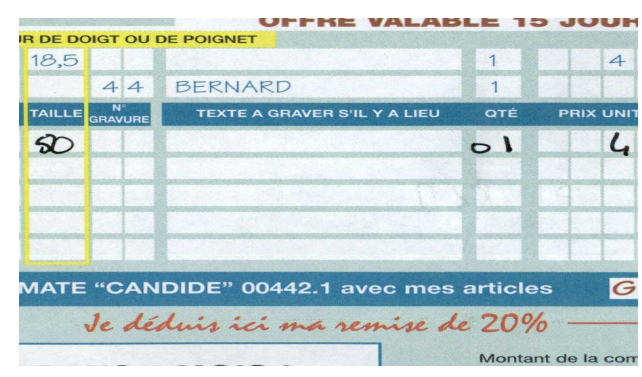

b) Original Image I 73727 different colors

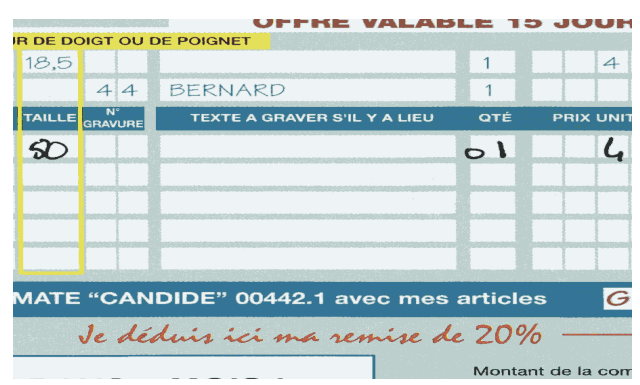

c) Fast Integral MeanShift image with 22colors

Figure 4: Fast Integral Mean Shift application.

\subsection{Segmentation of Thin Colored Objects by Using Color Morphology}

This step consists to generate a binary image of thin colored objects. It differs from the classical binarization of the luminance by an adaptive thresholding approach. For images which use colors having almost the same luminance, the thresholding of the luminance fails. We use the mathematical morphology because it can extract objects according to geometrical measures. Thin objects can be segmented by a classical Top-Hat transform. The closing of an image $I$ is the dilation followed by an erosion of the image with the same structural element (2). It enlarges the lighter foreground and removes the thin objects which are darker than the background (figure $5 \mathrm{c}$ ). The radius of the structural element $\mathrm{B}$ measures the thickness of the object to remove. The Black-Top-Hat (3) of an image I consists of make the difference between the closing image and the original image I, in order to highlight all thin colored objects which are darker than the background in luminance (figure 5d).

If we assume that all characters use colors which are darker than the background, in luminance, a BlackTop-Hat is efficient to extract all colored characters from any colored background. By using the interleaved bits order explained in (Chanussot et al., 1998) the multidimensional morphology in (RGB) color space can be substituted efficiently by a scalar classical morphology.

$$
\begin{aligned}
& \text { Closing }(\mathrm{I})=\operatorname{Erode}_{\mathrm{B}}\left(\text { Dilate }_{\mathrm{B}}(\mathrm{I})\right) \\
& \text { Black-Top-Hat }(\mathrm{I})=\text { Closing }(\mathrm{I})-\mathrm{I}
\end{aligned}
$$

To extract all colored characters even with fading ink, we apply an adaptive thresholding like Sauvola thresholding. In our case, we use the variable adaptive thresholding windows (Gaceb et al., 2013) which compute the best window size of the Sauvola 
thresholding for each pixel by using integral images (figure 5e).

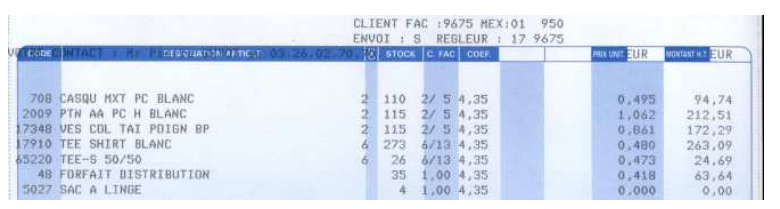

a) Original Image I

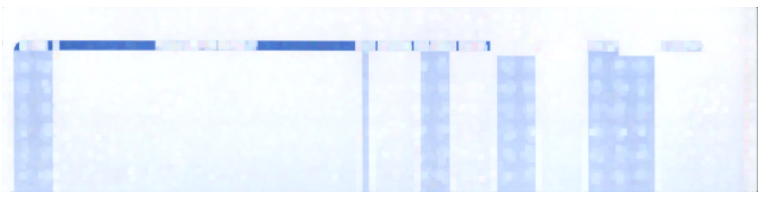

b) Dilation(I)

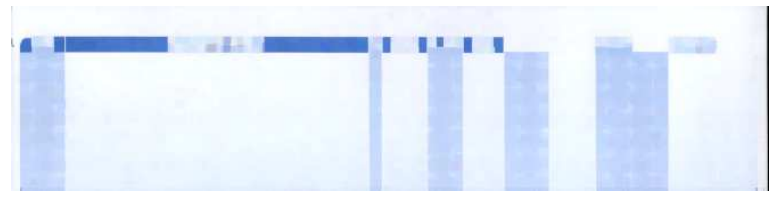

c) $\operatorname{Closing}(\mathrm{I})=\operatorname{Erode}(\operatorname{Dilate}(\mathrm{I}))$

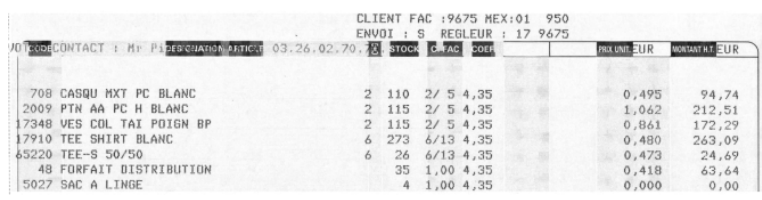

d) Black-Top-Hat(I) = Closing(I)-I

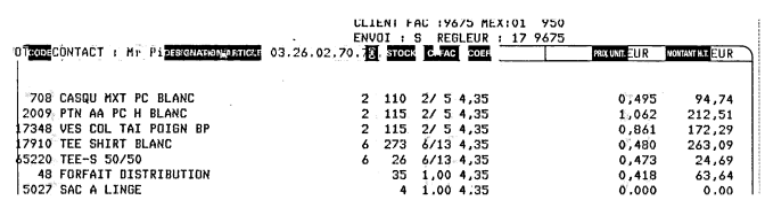

e) Adaptive Threshold of the Black-Top-Hat(I) by (Gaceb, 2013)

Figure 5: Black-Top-Hat Color Morphology operation

The difference between our thresholding and the classical Sauvola with fixed window size is noticeable on dithered images or images having noise and large objects. However, Sauvola thresholding can be used without significant loss of performance in the case of the segmentation of the Black-Top-Hat images because the size of objects cannot exceed twice the size of the structural elements. In this case the variable window size of the Sauvola thresholding (Gaceb et al., 2013) manages to reduce noise from the images.

In the domain of business documents, we cannot assume that text is darker than the background in luminance. Invoices and forms present inverted text which is brighter than the background. This inverted text is important for the recognition of business documents because it generally represents the labelling of a column or a row in a table. To extract inverted text, we must apply a White-Top-Hat which is the dual operation of the Black-Top-Hat transform. The opening of the image (4) deletes thin bright objects from darker background. The difference between the original image and the opening image (5) highlights the thin objects brighter than the background.

$$
\begin{aligned}
& \text { Opening }(\mathrm{I})=\text { Dilate }_{\mathrm{B}}\left(\operatorname{Erode}_{\mathrm{B}}(\mathrm{I})\right) \\
& \text { White-Top-Hat }(\mathrm{I})=\text { I-Opening }(\mathrm{I})
\end{aligned}
$$

However, we cannot combine the Black-Top-Hat and the White-Top-Hat results at pixel-level. The duality of these two morphological operations makes impossible the separation between inverted and noninverted text. In the Black-Top-Hat, inverted text becomes white over black background and in WhiteTop-Hat it appears black over white background. There is no simple binary operation which separate inverted and not inverted text. The local dominant colors bring a solution to segment simultaneously inverted and non-inverted text by morphology. This operation allows to measure the color of the background whether the text is inverted or not. The precision of the dominant color image is not important. In order to make a precise adjustment to the original image we dilate or erode the dominant color image with the original image. Among all possible existing methods, to compute the dominant color we have chosen the median filtering with a large radius since its complexity has been seriously reduced and can be calculated in constant time (Perreault, 2007) whatever the size of the window. Figure $6 \mathrm{~b}$ shows the result of the median with radius 15 or a window size of 30 on image in figure $6 \mathrm{a}$. The dominant color image is a coarse representation which we refine by applying a conditional erosion and dilation with the original image.

In classical morphology theory for binary image, the conditional dilation $\mathrm{CD}_{\mathrm{B}}{ }^{\mathrm{M}}(\mathrm{X})$ of a subset $\mathrm{X}$ by a structural element $\mathrm{B}$ conditioned to a mask $\mathrm{M}$ is equal to the intersection between the Dilated of $\mathrm{X}$ with the binary mask $M$ (6). Accordingly, the conditional erosion $\mathrm{CE}_{\mathrm{B}}{ }^{\mathrm{M}}(\mathrm{X})$ of a subset $\mathrm{X}$ by a structural element $\mathrm{B}$ conditioned to a mask $\mathrm{M}$ is equal to the intersection between the complementary of the erosion of $X$ with the binary mask $M(5)$.

$$
\begin{aligned}
& \mathrm{CD}_{\mathrm{B}}^{\mathrm{M}}(\mathrm{X})=\text { Dilate }(\mathrm{X}) \cap \mathrm{M} \\
& \mathrm{CE}_{\mathrm{B}}^{\mathrm{M}}(\mathrm{X})=(\text { Erode }(\mathrm{X}))^{\mathrm{C}} \cap \mathrm{M}
\end{aligned}
$$


Applied to greyscale images or to scalar color images by using interleaved bits of the three color channels, the conditional dilation $\mathrm{CD}_{\mathrm{B}}{ }^{\mathrm{M}}(\mathrm{X})$ of a scalar image $\mathrm{X}$ under condition of a scalar image $\mathrm{M}$ is equal to the minimum of the dilation of $\mathrm{X}$ and the image $M(8)$. By symmetry, the conditional erosion $\mathrm{CE}_{\mathrm{B}}^{\mathrm{M}}(\mathrm{X})$ is obtained by the maximum between $\mathrm{M}$ and the erosion of $\mathrm{X}(9)$.

$$
\begin{aligned}
& \mathrm{CD}_{\mathrm{B}}{ }^{\mathrm{M}}(\mathrm{X})=\min (\text { Dilate }(\mathrm{X}), \mathrm{M}) \\
& \mathrm{CE}_{\mathrm{B}}{ }^{\mathrm{M}}(\mathrm{X})=\max (\text { Erode }(\mathrm{X}), \mathrm{M})
\end{aligned}
$$

We detect and segment separately both inverted and non-inverted text by using the conditional dilation (11) and the conditional erosion of the dominant color image (12) restricted by the original image I, respectively.

$$
\begin{gathered}
\text { DominantLocalColor }(\mathrm{I}) \approx \operatorname{Median}(\mathrm{I}) \\
\mathrm{CD}_{\mathrm{B}}^{\mathrm{I}}(\operatorname{Median}(\mathrm{I}))=\min (\operatorname{Dilate}(\operatorname{Median}(\mathrm{I})), \mathrm{I}) \\
\mathrm{CE}_{\mathrm{B}}{ }^{\mathrm{I}}(\operatorname{Median}(\mathrm{I}))=\max (\operatorname{Erode}(\operatorname{Median}(\mathrm{I})), \mathrm{I})
\end{gathered}
$$

$\mathrm{CE}_{\mathrm{B}}{ }^{\mathrm{I}}($ Median(I)) erases all not inverted texts which are darker in luminance than the background (Figure 6c). Accordingly, $\mathrm{CD}_{\mathrm{B}}^{\mathrm{I}}(\mathrm{Median}(\mathrm{I}))$ deletes all inverted texts which are brighter than the background (figure $6 \mathrm{~d}$ ). To extract the not inverted text, we define Positive(I) by taking the difference between $\mathrm{CE}_{\mathrm{B}}{ }^{\mathrm{I}}$ (Median (I)) and the original image I (10). Accordingly, to segment inverted texts, we define Negative(I) by taking the difference between the original image $\mathrm{I}$ and $\mathrm{CD}_{\mathrm{B}}^{\mathrm{I}}(\operatorname{Median}(\mathrm{I}))(11)$.

$$
\begin{aligned}
& \operatorname{Positive}(\mathrm{I})=\mathrm{CE}_{\mathrm{B}}^{\mathrm{I}}(\operatorname{Median}(\mathrm{I}))-\mathrm{I} \\
& \text { Negative }(\mathrm{I})=\mathrm{I}-\mathrm{CD}_{\mathrm{B}}^{\mathrm{I}}(\operatorname{Median}(\mathrm{I}))
\end{aligned}
$$

Figure $6 \mathrm{e}$ and figure $6 \mathrm{f}$ show the positive and the negative parts of thin colored objects, respectively. Both positive and negative images contain a strong dithering of the background in the blue column (Figure $6 \mathrm{~h}$ ). The final result is obtained by taking the min of the adaptive thresholding of the positive and the negative image (figure $6 \mathrm{~g}$ ). The radius of the median in order to find the local dominant colors depends on the size of the text to segment.

The window size of the median operator must be larger than the maximum text height. For color images with resolution of $300 \mathrm{dpi}$ of resolution, the maximal height of text does not exceed 25 pixels.
The size of the window for the median filtering must be larger than this value. In order to have more margin, we chose a window size larger than 30 or a radius larger than 15 .

Figure $8 \mathrm{a}$ and $8 \mathrm{~b}$ show that AColDPS can process successive inclusion of color frames without heuristics. The dithering part of the blue column is also segmented like all thin objects such as characters and thin graphical objects. Invoices and forms frequently use dithered background colors that need cleaning. In the next step, we propose a straightforward process to remove both large graphic objects and noise without connected components extraction by using morphological geodesic operations.

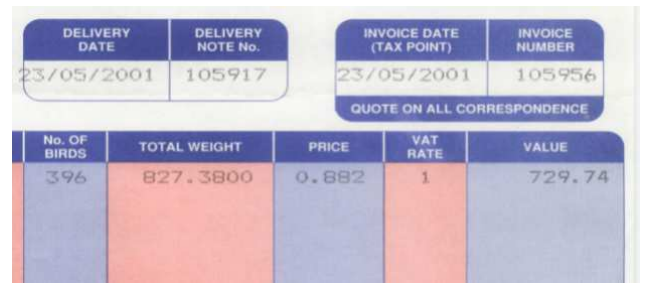

a) Original image $I$

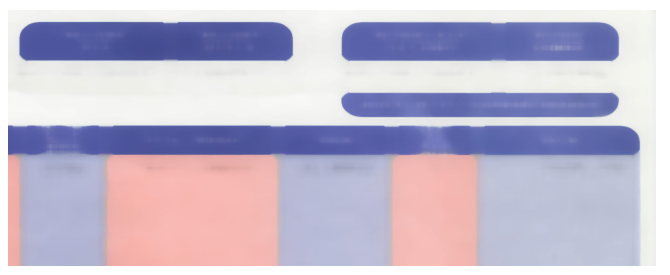

b) Median(I) filtering in constant time (perreault, 2007)

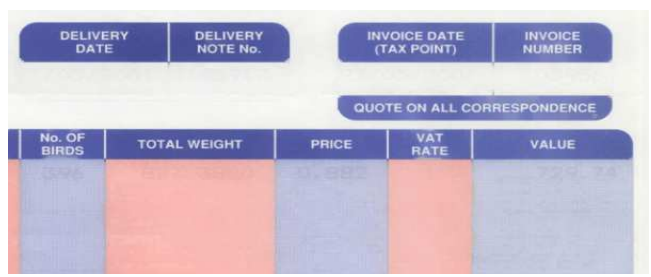

c) $\mathrm{CE}_{\mathrm{B}}{ }^{\mathrm{I}}(\operatorname{Median}(\mathrm{I}))=\max (\operatorname{Erode}(\operatorname{Median}(\mathrm{I})), \mathrm{I})$

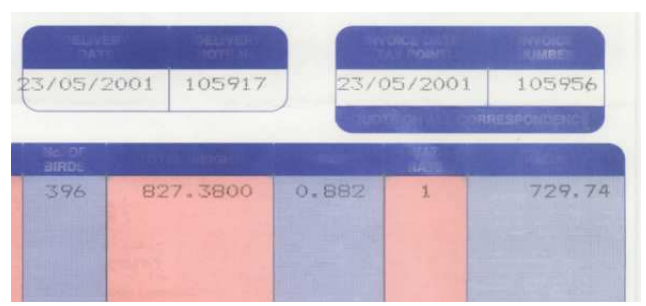

d) $\mathrm{CE}_{\mathrm{B}}^{\mathrm{I}}(\operatorname{Median}(\mathrm{I}))=\max (\operatorname{Erode}(\operatorname{Median}(\mathrm{I})), \mathrm{I})$ 


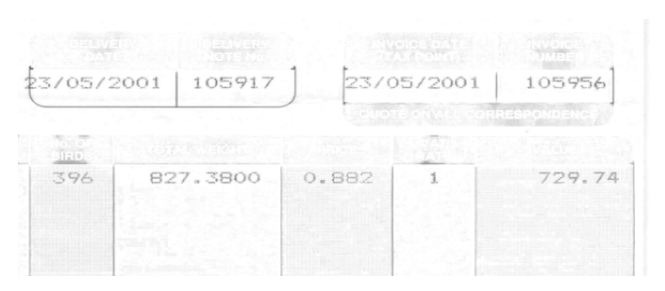

e)Positive $(\mathrm{I})=\mathrm{CE}_{\mathrm{B}}{ }^{\mathrm{I}}($ Median $(\mathrm{I}))$ - I shows not inverted texts

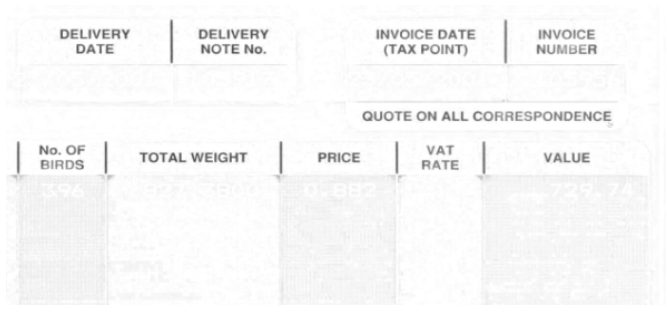

f)I - $\mathrm{CD}_{\mathrm{B}}{ }^{\mathrm{I}}(\operatorname{Median}(\mathrm{I}))$ shows inverted texts

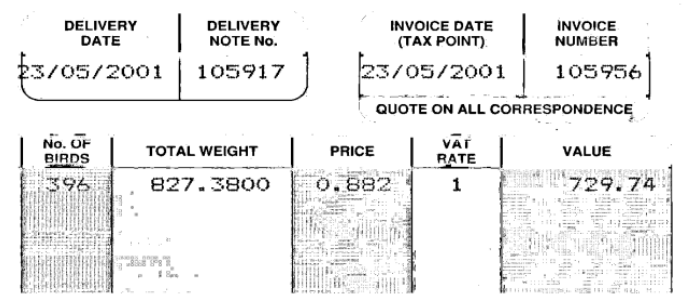

g) $\operatorname{Min}($ Threshold(Positive(I)),Threshold(Negative(I)))

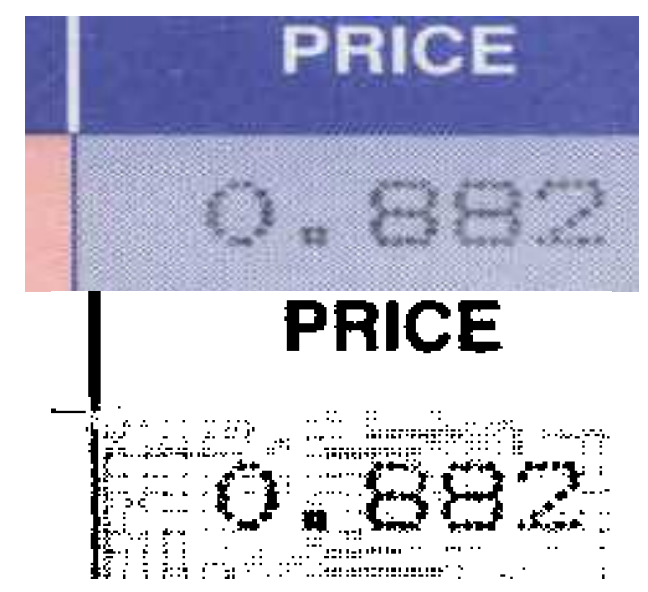

h) Zoom to show the dithering effects in the blue column

Figure 6 : The succession of operations to segment color images containing inverted and not inverted texts.

\subsection{Separation of Text from Graphics, Image Noise and Dithered Background}

This step does not use any connected components extraction or analysis. It is only based on morphological convolution for geodesic measures described in section 2 applied on the binary image obtained by the previous step.

\section{a) Despeckle}

To save computational time, we apply a despeckle to quickly remove noise and dithered background. We apply a two pas distance transform in the 8connectivity neighbourhood of the binary image. The maximal value of the distance transform is reached along the medial axis (Chassery,1991) and measure the minimum geodesic distance from each pixel to the contours of the object. The maximal value inside each object measures the maximal thickness of that object. To propagate the maximal thickness value inside each object, we repeat until the morphological convolution of dilation with a null mask MO is stable. All pixels inside each object will take the same value equal to the maximal thickness of that object. The despeckle consists to erase in one pass, all objects having a maximal thickness of Thicknessmin threshold. It is similar to threshold the resulting image with a Thicknessmin threshold. Figure 7 shows a despeckle of document figure 6 with ThicknessMin=2.

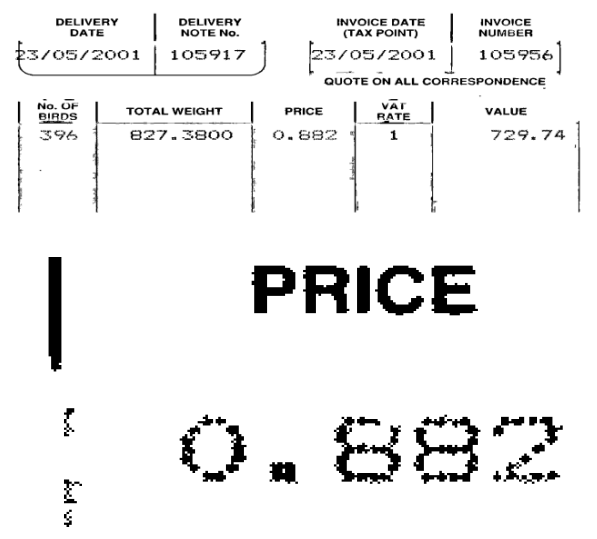

Figure 7: Results of the Despeckle by morphology

\section{b) Separation between Graphics/Text/Dithered Parts}

The geodesic measure of width and height is not the measure of the bounding box surrounding the object, but the measure of the width and height inside the object. The geodesic measures bring more pertinent information than classical spatial measures. Moreover, the noise removal based on geodesic width and height has nothing to do with the morphological despeckle described previously based on the objects thickness. The Objects' thickness and geodesic width and height are complementary 
information for noise and dithered background removal. Our separation is based on the geodesic width and height of binary objects calculated by morphological convolution. We repeat the morphological convolution of dilation with the Feret mask FERET9O and FERETO for $90^{\circ}$ and $0^{\circ}$ direction on the binary image, respectively, until there is no change. It provides two images GeodesicHeight / GeodesicWidth with value 1 on the bottom/left outermost points of each objects and the geodesic width and height on the top/right outermost points of each objects (figure $8 \mathrm{c}, 8 \mathrm{~d}$ ), respectively. To propagate the maximal geodesic width and height values inside each object, we repeat until the morphological convolution of dilation with a null mask NULL MOis stable. All pixels inside each object contain the geodesic width and height of the object (figure 8e, 8f). These images will be useful for despeckle, noise and dithered part removal, suppression of graphics and the separation of characters connected to graphics described in the next step.

To remove noises and speckles generated by the dithered backgrounds, we shift pixel $(x, y)$ from the binary image to the image speckles Image if both GeodesicHeight/ GeodesicWidth images have a value strictly inferior to HeightMin and WidthMin pixels respectively. All objects from the binary image having a geodesic height and width which do not exceed HeightMax and WidthMax respectively, are shifted in the image Texts Image because they have the size to be potentially characters. All the other objects are classified into the image Graphics Image if the geodesic width or heights exceed WidthMax or HeightMax, respectively. They represent large objects that we consider as graphics (Algorithm 1).

Algorithm 1: separation between graphics/text/dithering

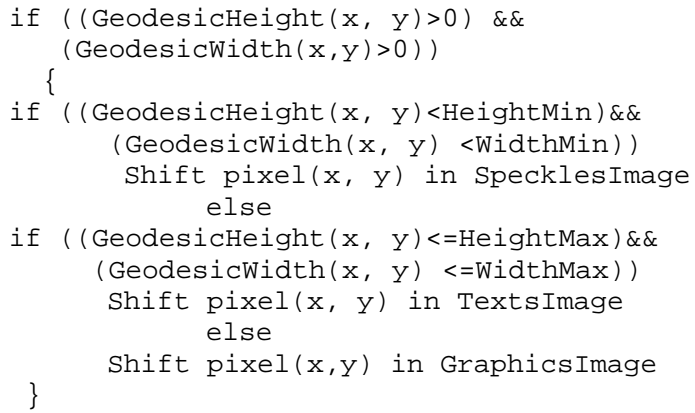

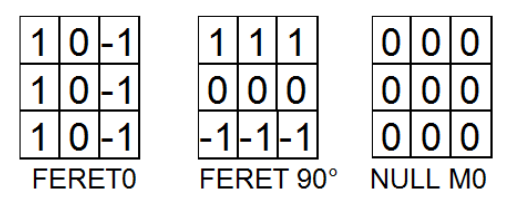

We choose to set HeightMin and WidthMin to the value 3 in order to keep and thin characters like 'I' or '1'. For printing documents, we set WidthMax and HeightMax to value 64 for 300dpi images, because characters cannot exceed this size. For ligatured manuscripts we set WidthMax and HeightMax to value 512 and 128 respectively, in order to shift correctly handwritten words in the image TextsImage.

We keep the graphical information into a binary image for further analysis to localise frames and detect tables in future work. Tables or frames bordered by dot lines or dashed lines cannot be separated from the text image because the geodesic width or height are similar to the size of characters.

Figure 9 illustrates the separation between speckles, text and graphics. Figure 9a shows the difficulties of color printed invoices with complex color background. Figure $9 \mathrm{~b}$ shows the large amount of speckles (noise and dots from dithering part) deleted from the binary image. Figure 9c displays the graphics elements and large objects. We notice that small vertical lines from tables in columns are not classified into graphics because their heights do not exceed HeightMax.

The horizontal dot line and other small components from the graphical background are classified to the image TextsImage instead of Graphics Image because their geodesic widths are under the limit WidthMax. These errors have no consequences, because during the layout analysis, these random elements from the image TextsImage will be rejected because they are not aligned enough to build a text line.

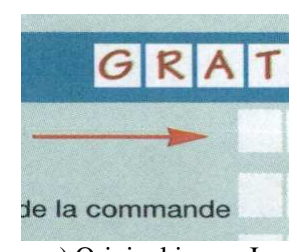

a) Original image I

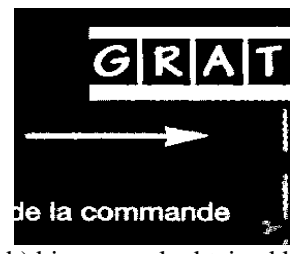

b) binary mask obtained by the color segmentation step 


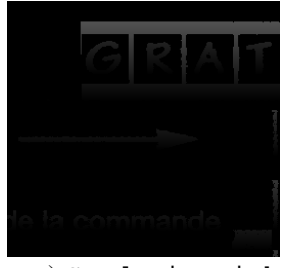

c ) GeodesicHeight Obtained by morphological withFERETOand FERET90

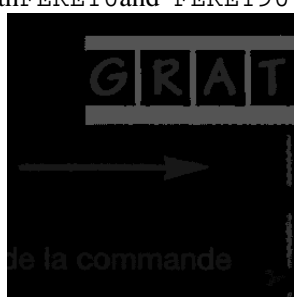

e)GeodesicHeight

after propagation of maximal values by morphological convolution of dilation with NULL MO mask.

Figure 8: Results of the geodesic transform by morphological convolution
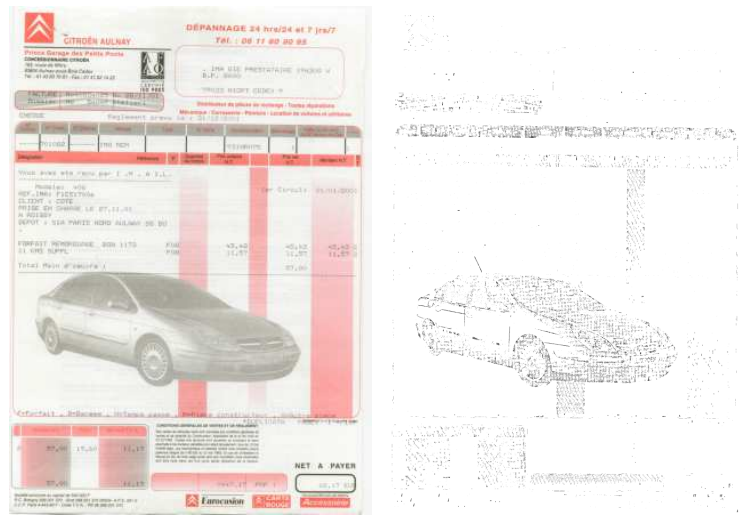

a) Original Image I

b) Speckles Image

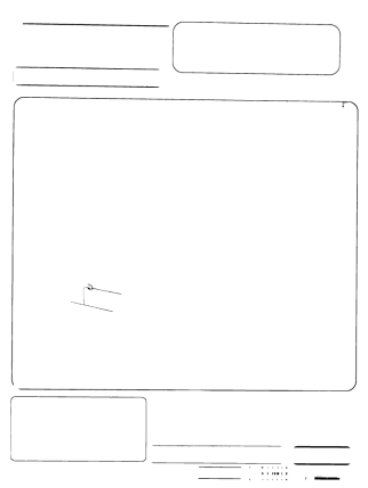

c) Graphics Image

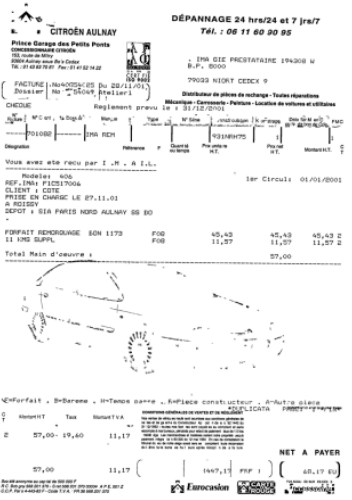

d) Texts Image
Figure 9: separation between noise/graphics/text
We design the system to be sure that all the characters are correctly classified into the image Texts Image. But for characters connected to the graphical elements, these characters are found in the Graphics Image. The next step aims to separate characters connected to graphics.

\subsection{Separation of Characters Connected to Graphics}

Figure 10 illustrates frequent cases for invoices with printing text which overlap lines surrounding frames or tables. When colors cannot separate characters and graphics, the image Graphics Image contains textual element which touch graphics (Figure 10b). We can achieve a coarse separation between characters and graphics by using elementary morphological operation with the existing information provided by the system. The binary morphological closing of Graphics Image, with an horizontal / vertical element $\mathrm{Bh} / \mathrm{Bv}$ respectively, removes all characters that touch graphics both vertically / horizontally (figure $10 \mathrm{c}, 10 \mathrm{~d}$ ). We define H_Text (15) and V_Text(16) by the difference between the horizontal / vertical closing of GraphicsImage with the image GraphicsImage itself, respectively. Images H_Text/V_Text show characters disconnected with horizontal / vertical lines (Figure 10e, 10f), respectively.

$$
\begin{aligned}
& \text { X= Graphics Image } \\
& \text { H_Text=HorizontalClosing }{ }_{\mathrm{Bh}}(\mathrm{X})^{\mathrm{c}} \cap \mathrm{X} \\
& \quad \mathrm{X}=\text { Graphics Image } \\
& \text { V_Text=VerticalClosing }{ }_{\mathrm{Bv}}(\mathrm{X})^{\mathrm{c}} \cap \mathrm{X}
\end{aligned}
$$

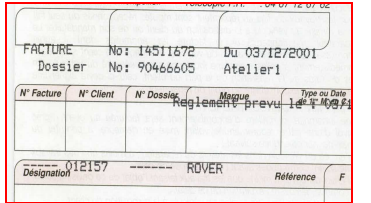

a) Original Image with overlappe characters touching graphics

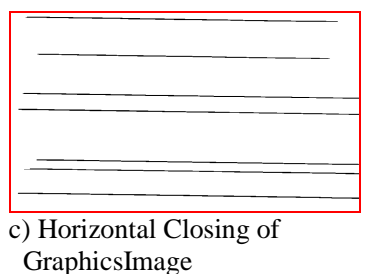

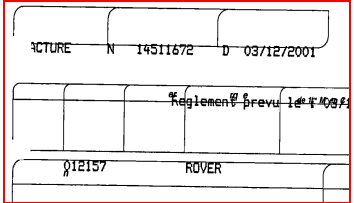

b) GraphicsImage

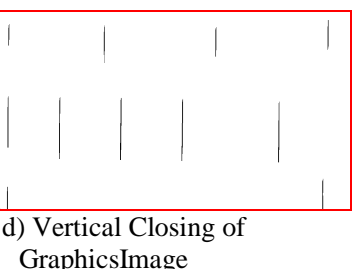

d) Vertical Closing of GraphicsImage 


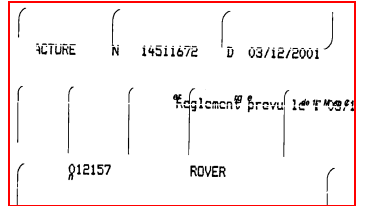

e) H_Text (7)

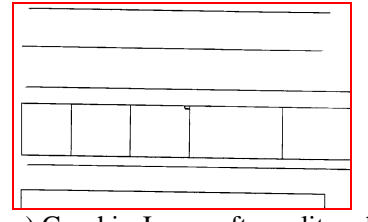

g) GraphicsImage after split

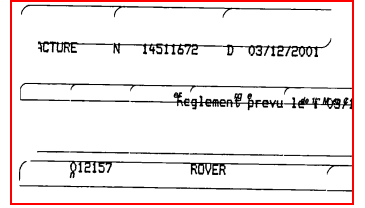

f) V_Text (8)

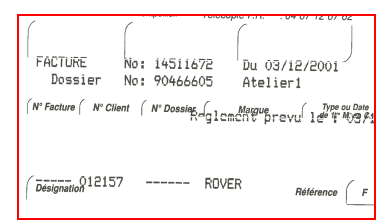

h) Text separated from graphics
Figure 10: Separation of characters connected to graphics

The size of the horizontal and vertical structural elements $\mathrm{Bh}$ and $\mathrm{Bv}$ must be fixed to the minimal width and height of characters to separate from the graphics, respectively. Typically we use WidthMax and HeightMax for the size of the horizontal and vertical structural elements respectively.

The reconstruction of characters after splitting them from a graphic line is possible but we do not achieve this restoration. This character reconstruction will be detailed in future work. Our proposed morphological operation works perfectly well for text touching straight lines only, and is also tolerant to image skew (figure 10h).

\subsection{Color Fusion and Selection}

This step consists of combining the color information from the MeanShift clustering and the color segmentation by morphology to merge outlier colors classes to the main color classes. This step also ranks the text color layer by frequency and selects the main text colors. We have already illustrated (figure 4) that the MeanShift produces classes of outliers colors due to the existing color transitions along characters contour. As the Meanshift is applied only in the colorimetric space, for each pixel independently to the other neighbouring pixels, these color outliers cannot be avoided. We have tested several pre-processing algorithms such as the edge preserving smoothing (Nikolaou,2009) or other choc filters to suppress the color transitions along contours. However, this kind of pre-processing is time consuming and cannot be used for real time applications.

We have developed a straightforward merging process which takes into account the spatial cooccurrence of colors classes in the segmented image by the MeanShift. We use Texts Image to compute statistics about the connectivity of colors classes found by the MeanShift. We only focus on text color because the color of the background is useless for our application. In the image Texts Image, we compute the $2 \mathrm{D}$ spatial co-occurrence $\mathrm{H} 2 \mathrm{D}(i, j)$ equal to the number of class color $i$ connected spatially to class color $j$ in all the inside characters of the imageTexts Image. We use a 8-connectivity to count correctly in one pass $\mathrm{H} 2 \mathrm{D}(i, j)$. We compute H1D ( $i$ ) the number of occurrence of the class color from $\quad H 2 D(i, j) . \quad C(i, j)=H 2 D(i, j) / H 1 D(j)$ measures the degree of connectivity between the class colori with the class color $j$. Color Outliers share a high connectivity with main colors of characters.

Algorithm 2 merges color class $i$ to color class $j$ if the connectivity $C(i, j)$ exceeds $\mathrm{Cmin}=0.25$ and if $C(i, j)$ and $H 1 D(j)$ are maximal and the colorimetric distance is minimal. To keep the color coherency, we merge small class to large class and not the inverse.

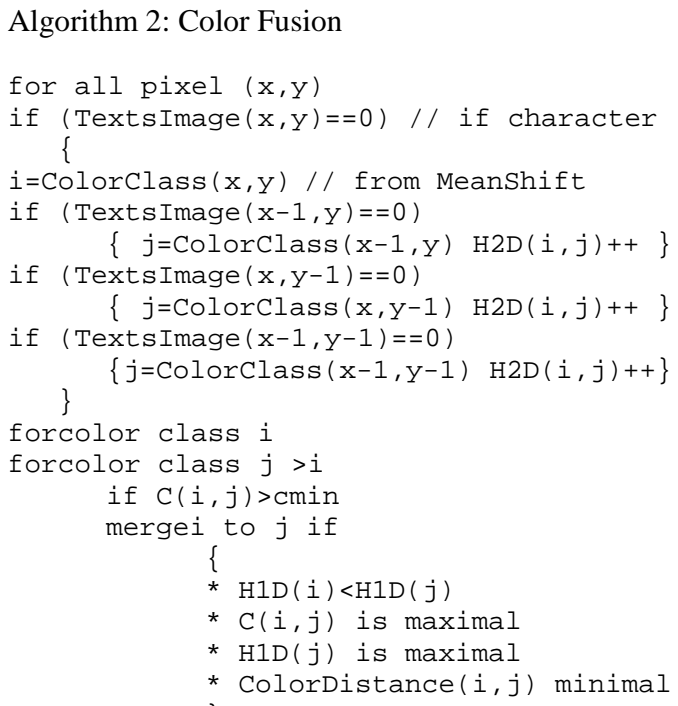

After merging the color classes in Colorclass, we repeat algorithm 2 until there is no more changes. The iterations are necessary to merge successively layers of colors around characters contours. To select the right number of different text color, we rank the text color classes in descending order of frequency. There is a gap between two consecutive ranked color classes of the decreasing curve of frequencies. We set the number of text colors in the middle of the larger gap between successive color classes. 


\subsection{Algorithmic Optimization}

Several hundreds of thousands of documents are automatically processed daily by the company. We must reduce the algorithmic complexity of each step of the AColDPS system. The overall processing for each image must not exceed few seconds without parallelization. The objective consists of computing an image in less than a second with parallelisation.

The color clustering is already optimized with a complexity of a $\mathrm{O}(\mathrm{N})$ by using the Fast Integral MeanShift based on integral cubes (Lebourgeois et al., 2013). The adpative thresholding of the color morphology results is also already optimized with integral images (Gaceb et al., 2013). Median filter is also computed in constant time by using partial histograms correspondence of sliding windows between two successive windows (Perreault, 2007). We have speeded-up the median filtering by applying it on a low resolution image for a degraded result. However, the median result is just a coarse representation of the local dominant color we use as seeds for a conditional dilation or erosion. The quality of the median result is not taken into account during the process. For the morphological color operation of dilation and erosion, integral images cannot be used with min and max operators, only summation of functions can be computed. To optimize the color morphological operations of dilation and erosion, we use the same idea from (Perreault, 2007). Instead of computing the statistical histogram to calculate the median values, we compute the local maxima and minima of a sliding window by using an horizontal raw vector which stores the maximal/minimal values of each column of the windows. When the window slides from a pixel to another, we just shift the values in the row vector and compute the maxima/minima of the new column that we compare to the maxima/minima of the row horizontal vector only once.

Color segmentation and the clustering process run in much reduced time and depend only on the number of pixels to process. The color fusion and selection step depends on the number of colors found by the fast integral MeanShift. As the number of clusters is reduced, the number of operations are not time consuming. The computational time for the separation between text from graphics and noise is not predictable. The morphological convolution like all geodesic transformation is repeated until there is not change. The number of iterations depends on the complexity of shapes and the alignment of dots for the dithered image. The more the image contains complex graphics and noise or dithered background, the more time this step will take. The time of the processing is maximal for complex shapes like Peano curves or other space-filling curve. For a 300dpi image 1640 x 2332, it takes less than 3 sec. for color clustering, $6 \mathrm{sec}$. for color image segmentation by morphology and colors fusion and selection on one core without parallelization. The separation of text from graphics, image noise and dithered background can take less than a second for simple and clean image to several seconds for complex images with dithered background. All the algorithms are sequential and can be easily parallelized.

\section{RESULTS}

\subsection{Global Performances}

We first illustrate the performances of the color automatic segmentation on a test image provided by the private company Janich\&KlassComputertechnik $\mathrm{GmbH}$ which has developed the software DpuScan and its Advanced Color Document Processing (ACDP) tool, which is widely used in the industry and distributed worldwide. DpuScan is well-known to be the best tool to separate text colors in business documents. But it is achieved manually by selecting each color background and text color.

Figure 11 shows that AColDPS find text color automatically and achieve a good segmentation of this image. We display all color layers of text in the same image with an artificial white background to save place. Figure 12 and 13 show the pertinence to use color information to segment text from complex pre-printed backgrounds. The thresholding of the luminance image (figure 12b) make difficult the separation between handwritten text and the preprinted forms. After a color analysis, the added handwritten text can be easily segmented. Figure 14 zoom on the table headings of the figure 4. It illustrates the color separation between overlapped texts in the worst case when inverted text crosses non inverted text. AColDPS separates correctly the two layers of text (figure 14).
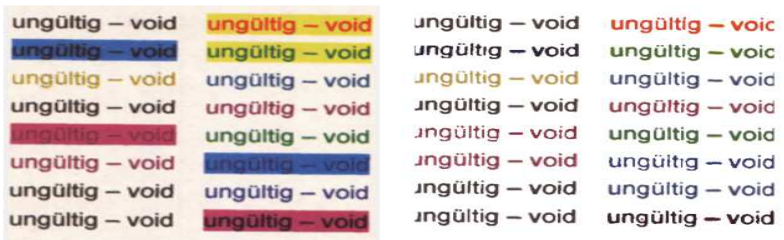

Figure 11: AColDPS result on DPUscan test image. 


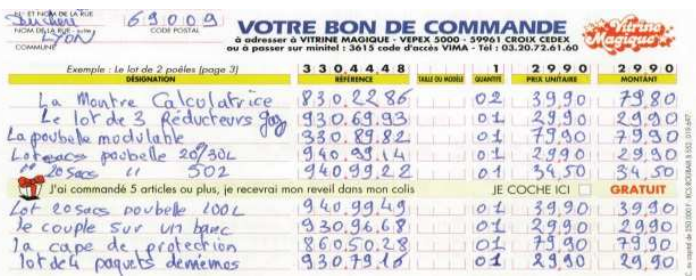

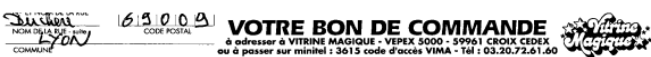

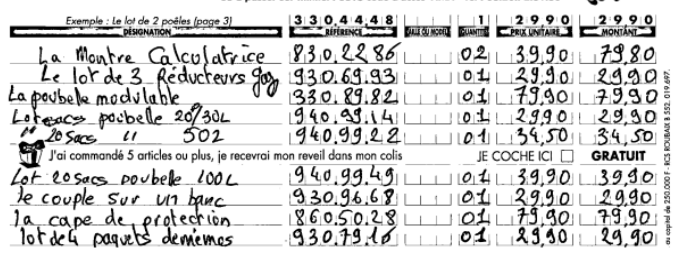

a) Binarization of the luminance (Sauvola)

Duchere, 69009 VOTRE BON DE COMMANDE
LYON,

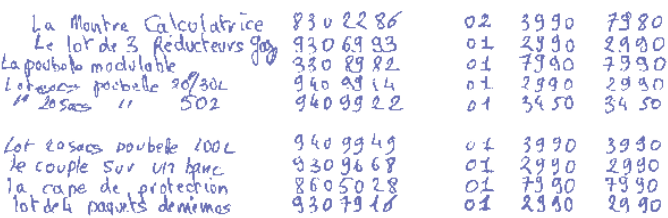

b) Main text colors (handwritten text added to the form)

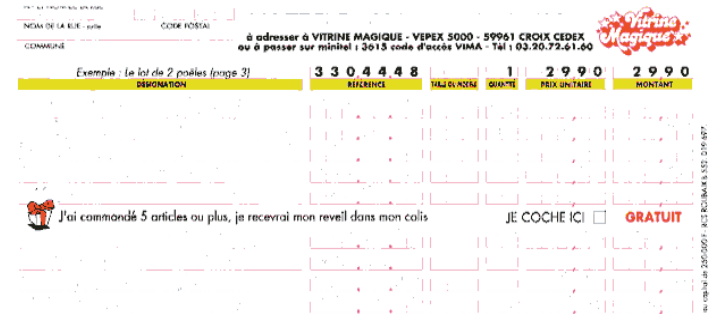

c) 3 other color layers combined together (the pre-printed form)

Figure 12: Color segmentation outperforms the adaptive thresholding of the luminance for character segmentation.

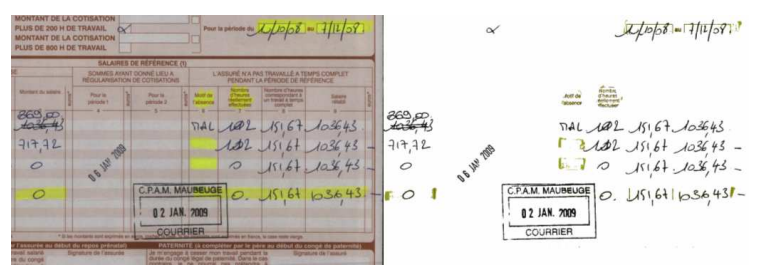

Figure 13: AColDPS separates correctly color handwritten text and the background even with highlighting regions.

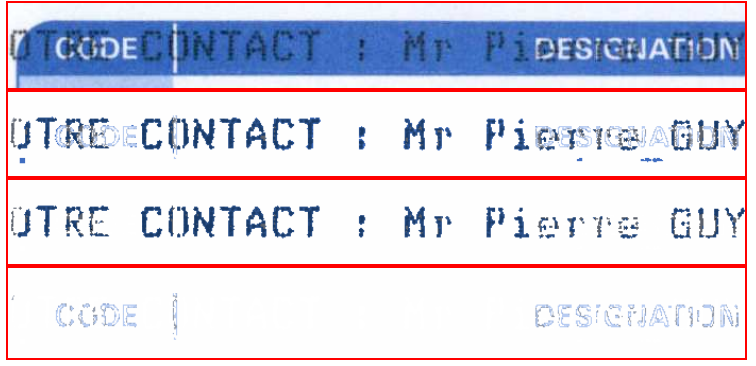

Figure 14: Correct color separations with overlapped inverted and not inverted texts.

\subsection{Evaluation on the Database}

We have tested the proposed system on 529 color images of various invoices and forms in real situation. Among 529 images we manually found 4 images with some problems of segmentation. Among these errors, we found 2 images that present a change of text color, detected by the system, because of the ink bleed trough of the color background to the characters of the foreground (Figure15). 1 images show handwritten texts totally illegible because of the ink fading. 1 error is due to the printing of black characters crossing a large black frame. Most of errors can be explained by the quality of the document itself. We have achieved $99.25 \%$ of correctly segmented document.

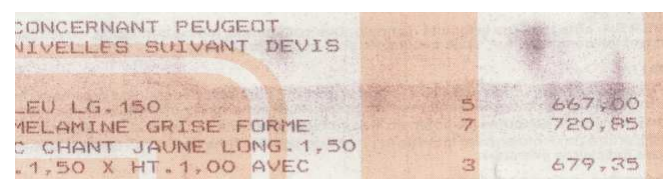

Figure 15 Failure of AColDPS because of color transfer from the background to the foreground.
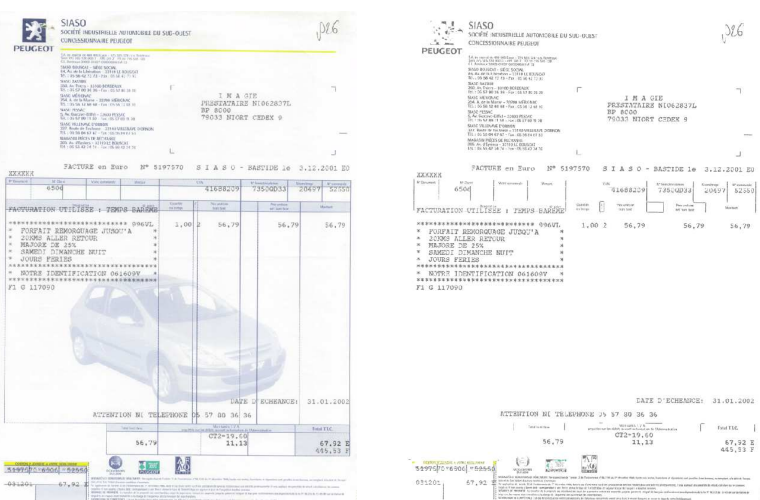

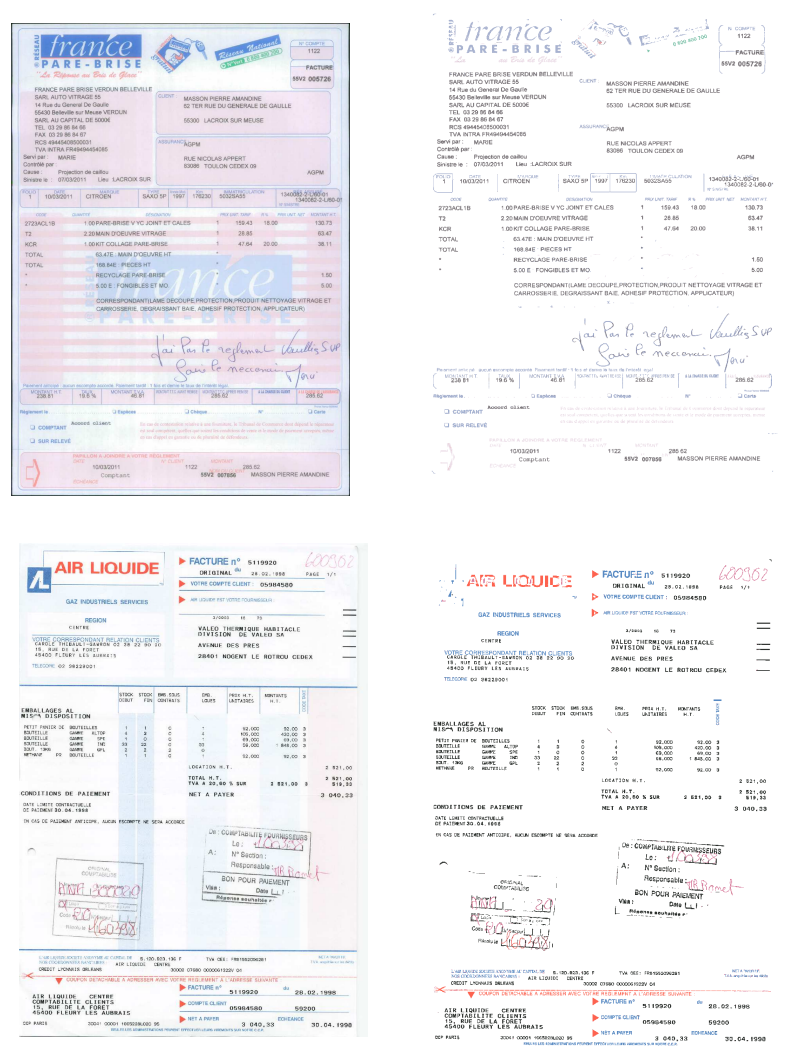

Figure 16: Successes of AColDPS

\section{CONCLUSIONS}

In this paper, we have presented an unsupervised fully automatic system for color business document segmentation. We have developed the first fully data-driven pixel-based approach that does not need a priori information, training or manual assistance. The proposed method has the following advantages: 1) It does not require any connected component analysis and simplifies the extraction of the layout and the recognition step undertaken by the OCR; 2) it processes inverted and non-inverted text automatically, using color morphology, even in cases where there are overlaps between the two; 3 ) it efficiently removes noise and speckles from dithered background and automatically suppresses graphical elements using geodesic measurements; 4) it splits overlapped characters and separates characters from graphics if they have different colors. The proposed Automatic Color Document Processing System has the potential to be adapted into different business document images. The system outperformed the classical approach that uses binarization of the greyscale image and simplifies both the extraction of the layout and the recognition performed by the OCR. In future works, we plan to find a solution to reconstruct characters crossing graphical elements.

\section{ACKNOWLEDGMENT}

This work is granted by ITESOFT for the project DOD.

\section{REFERENCES}

L. Bottou ,P. Haffner, PG. Howard, Y. LeCun, Djvu: analyzing and compressing scanned documents for internet distribution. ICDAR, 2001.

K. Jung, J. Han, Hybrid approach to efficient text extraction in complex color images, PRL, V. 25, I. 6, 19 April 2004, Pages 679-699.

D. Karatzas, et al., Color text segmentation in web images based onhuman perception Image and Vision Computing, Volume 25, Issue 5, 1 May 2007, Pages 564-577

E. Badekas et al., Text segmentation in color documents, IJIST, V. 16, I. 6, 2006, Pages: 262-274.

Y. Peng, J. Xiao, Color-based clustering for text detection and extraction in image, ICM 2007, Pages 847-850.

A. Ouji, et al., Chromatic /achromatic separation in noisy document images, ICDAR 2011.

A. Ait Younes et al., Color Image Profiling Using Fuzzy Sets, TJEECS, 13(3):343-369, 2005.

E. Carel et al., Dominant Color Segmentation of Administrative Document Images by Hierarchical Clustering, DocEng 2013.

S.Perreault and Patrick Hebert, Median Filtering in Constant Time, IEEE Image Processing 2007.

J. Chanussot \& P. Lambert, "Total ordering based on space filling curves for multivalued morphology", Proc. ISMM'98, June 1998, pp 51-58.

Jean Serra, Image Analysis and Mathematical Morphology, Academic Press, London, 1982.

E.Aptoula et al., Multivariate mathematical morphology applied to color image analysis. In Chapter 10: Multivariate Image Processing, 2009.

P. Soille, Morphological image analysis: Principles and applications. In Springer, 2004. 2nd Edition.

Comaniciu et al., 2002, MeanShift: A Robust Approach toward Feature Space Analysis, PAMI, Vol. 24, No. 5.

Gaceb D., et al., Adaptative Smart-Segmentation Method for Images of Business Documents, ICDAR 2013, pp. 118-122. .

F. Lebourgeois et al., Fast Integral MeanShift: Application to Color Segmentation of Document Images, ICDAR 2013, IEEE ed. Washington, USA. pp. 52-56. 